\title{
Total Internal Reflection with Fluorescence Correlation Spectroscopy
}

Nancy Thompson

University of North Carolina, Chapel Hill, NC

The combination of total internal reflection illumination with fluorescence correlation spectroscopy (TIR-FCS) allows one to examine in quantitative detail a variety of biophysical properties related to the motions and interactions of fluorescent molecules near the interface of a transparent planar surface and an adjacent solution. Several experimental and theoretical aspects of this combination will be discussed.

TIR-FCS has allowed characterization of local diffusion coefficients and concentrations of fluorescently labelled antibodies in solution but very close to substrate-supported phospholipid bilayers. TIR-FCS has also been used to examine the interaction kinetics of fluorescently labelled mouse IgG specifically and reversibly associating with the mouse receptor Fc $\gamma$ RII, which was purified and reconstituted into substrate-supported planar membranes.

This method also has the potential, through the use of a single fluorescent reporter, of providing information about the thermodynamics/kinetics of non-fluorescent molecules which participate in surface binding mechanisms; e.g., those that compete with fluorescent reporters for surfaceimmobilized receptors or those that interact on the surface with the receptors and reduce or enhance the interaction of the fluorescent reporters with the surface binding sites. 